\title{
Propagation of Stall in a Compressor Blade Row
}

\author{
FRANK E. MARBLE $\dagger$ \\ California Instilute of Technology
}

\begin{abstract}
Recent experimental observations on compressors, in particular those of Rannie and Iura, have clarified some features of the phenomenon of stall propagation. Using these observations as a guide, the process of stall in an airfoil cascade has been characterized by a static pressure loss across the cascade which increases discontinuously at the stall angle, the turning angle being affected in only a minor way. Deductions from this simple model yield the essential features of stall propagation such as dependence of the extent of stalled region upon operating conditions, the pressure loss associated with stall, and the angular velocity of stall propagation. Using two-dimensional approximation for a stationary or rotating blade row, free from interference of adjacent blade rows, extent of the stalled region, the total pressure loss and stall propagation speed are discussed in detail for a general cascade characteristic. Employing these results, the effect of stall propagation upon the performance of a singlestage axial compressor is illustrated and the mechanism of entering the regime of stall propagation is discussed. The essential points of the results seem to agree with experimental evidence.
\end{abstract}

\section{INTRODUCTION}

$\mathbf{I}$ THE RATE AT WHICH FLUID passes through a compressor blade row is decreased while the rotative speed remains constant, the angles of attack of the compressor blades eventually exceed that for which unseparated flow is possible. Individual blades then behave in a manner somewhat resembling stalled isolated airfoils and the compressor is said to stall. The nature of compressor operation in the stall regime is of considerable importance not only because of the attending loss in efficiency and pressure rise, but also due to the fact that the onset of stall is accompanied by pulsation and oscillating blade loads which may lead to structural failure of the blades. The complexity and general irregularity of the flow during stalled operation have, in the past, discouraged a detailed study. Recently several experimental investigations have demonstrated some regularities in the process. It appears that the blades of a given blade row do not stall simultaneously, but initially only a few blades stall. Furthermore, it is observed that the stalled region of a blade row does not always consist of the same group of blades but that the region moves about the blade row at an appreciable fraction of the rotor velocity. The name stall propagation or rotating stall originates from this behavior. In addition to the reasons mentioned above, stall propagation is of impor-

Received April 7, 1954.

* This work was performed, in part, under the financial support of the Office of Scientific Research, United States Air Force.

$\dagger$ Associate Professor. Daniel and Florence Guggeriheim Jet Propulsion Center. tance through its possible connection with compressor surge and because the appearance of the more or less regular phenomenon may indicate that the compressor is operating near its surge line.

During the past few years experimental observations at several research institutions and manufacturing establishments have indicated stall propagation in a variety of compressors. Unfortunately, most of this information exists only in private communications. It is probable, however, that the regularity was first recognized by Emmons together with his co-workers at Harvard University, and some of their findings have recently been reported in reference 1 . Of the available experimental results concerning stall propagation, the most detailed and complete are those of Rannie and Iura, ${ }^{2}$ performed at the California Institute of Technology on a large three-stage axial compressor. Knowledge of these experiments has been the principal guide in the present formulation of the problem.

The results of Rannie and Iura indicate that two types of stall propagation occur which are, at least superficially, different; in one the stall covers the entire blade length, in the other only a portion of the blade length. As the compressor is throttled, a single stalled area is observed which covers only the root or tip regions of a few blades. With further throttling two, three, or more similar regions appear, uniformly spaced about the periphery. The propagation speed remains remarkably constant as throttling proceeds. Finally, with further decrease in flow, the pattern changes to a single region extending over the blade length; this change is accompanied by a small decrease in propagating speed. This type of pattern persists until the flow is nearly shut off, the peripheral extent of the stalled region increasing as the flow is reduced. Some information on experiences with other machines has been reported by Huppert and Benser. ${ }^{3}$

The basic features of the stall propagation phenomenon seem to be quite clear. When the blade row operates at nearly its stall angle, any small disturbance will cause one or more of the blades to stall. The stall reduces the blade force and decreases the local pressure rise across the blade row so that the flow will be retarded near the stalled region and diverted to either side. Depending upon the geometry of the blade row, the flow angles upstream of the blade row will be increased on one side and decreased on the other. Where the flow angles increase, neighboring blades stall and the stalled region propagates in that direction. Where flow angles are decreased, blades already 
stalled may be relieved so that the stalled area retains a definite length. The rate of propagation depends upon some characteristic time associated with the stalling process. This characteristic time is related to the process of boundary-layer separation and establishment of a "stalled" flow pattern from an unstalled flow pattern. Some further results of Rannie and Iura clarify this point. They observed that the removal of every second blade from one or more of the blade rows had only a slight effect upon the speed of stall propagation. Consequently the influence of time required for boundary-layer separation must be rather small, since the propagating speed would be inversely proportional to the blade number if this were the governing phenomenon. Therefore the speed of propagation must be associated with the time required for the existing pressure difference across the blade row to transform the flow pattern. This time is not strongly dependent upon the blade geometry or the blade gap. On this basis an elementary model for the stalling process was constructed. Then it was not a difficult matter to deduce the resulting details of stall propagation.

It is a pleasure for the author to express his deep gratitude to Professor H. S. Tsien who supplied the impetus to start the investigation and the encouragement to finish it. The initial formulation of the problem and the requirements of the model for the stall process were suggested by Dr. Tsien. Professor W. D. Rannie likewise contributed greatly to the present picture of stall propagation through illuminating discussion of his experimental work. It was Dr. Rannie who first pointed out that considerable clarity could be gained by departing from the conventional formtilation according to airfoil theory of cascades. Professor W. R. Sears, Cornell University, has been most helpful; first in communicating the results of his analysis prior to publication, and later through stimulating discussion.

\section{The Physical Mechanism of Stall Propagation}

An airfoil exhibits two modes of operation: the normal mode, many features of which are well described by the theory of ideal fluids, and the stalled mode which may be described roughly by the ideal flow about a boundary consisting of a portion of the airfoil and a constant pressure stream line that bounds the stagnant wake. The pressure associated with the stream line is, in this case, equal to the undisturbed static pressure of the free stream. When airfoils are arranged in a cascade or lattice, as they are in a compressor blade row, they possess similar normal and stalled modes of flow. In a compressor cascade, the blades act to reduce the flow velocity from upstream to downstream so that for the normal mode of operation a static pressure rise occurs across the blade row. Thus when one airfoil of a cascade is stalled for some reason, the situation is somewhat different from that of an isolated airfoil because now the pressure associated with the free stream line is that far downstream of the cascade which is considerably in excess of that upstream. Since the high pressure now penetrates to a point near the leading edge of the stalled airfoil, the pressure in the entire vicinity is increased. As a consequence the velocity approaching the channel formed by the stalled surface and the next blade is reduced, as shown in Fig. 1, and the quantity of fluid passing through the channel is proportionately decreased.

Now if, during normal operation of the entire cascade, one of the airfoils stalls, the flow pattern corresponding to stall develops through intermediate stages indicated in Fig. 2. As the separation point moves forward from the trailing edge, the wake begins to develop and spreads downstream with a velocity less than the local free-stream velocity. The growth of the stall region gradually reduces the flow through the passage by diverting the fluid ahead of the cascade. The time elapsed during transition from unstalled to stalled velocity fields consists of that needed to separate the boundary layer and that required for the existing pressure difference across the cascade to deform the flow pattern. The latter is a simple inertial effect, while the boundary-layer separation is more complicated; actually the processes are not strictly independent.

The mechanism of stall propagation may be seen by observing, from Fig. 2, that, during successive stages in development of stall for one airfoil, the angle of attack on the airfoil just above it is increased. When this airfoil reaches its critical angle of attack, it too stalls and the process continues as shown in Fig. 3. Just how soon the adjacent airfoil begins to stall depends upon the normal angle of attack and the cascade geometry as well as upon the rate with which the neighboring flow field is modified by the airfoil originally stalled. Furthermore, the angle of attack is decreased upon the airfoils below the stalled one. Therefore as the stall region grows upward, airfoils far below will eventually become unstalled and the process will settle down to a steady propagating stalled region of constant length. The actual length depends, in particular, upon the proximity of the normal angle of attack to the stall angle.

The time required for the stall of one blade to deform the flow sufficiently to stall the next airfoil is a most significant item, and the factors that affect it may be isolated by an approximate analysis of dynamic equilibrium of the flow while the airfoil is stalling. Suppose that during normal operation a static pressure rise $\Delta p$ takes place across the blade row. If the pressure rise is small, it is related to the change $\Delta w$ in velocity from inlet to outlet by $\Delta p=-\rho w \Delta w$. Now when the blade stalls, the fluid passes through the stalled blade channel with negligible rise in static pressure. Therefore, assuming the static pressure field downstream of the blade row to be only slightly affected by the stall, a pressure difference $\Delta p$ is available for retarding the fluid which approaches from far upstream to flow through the stalled blade channel. Since the blade chord is neglected, the only characteristic length in the problem is the gap $g$ (or length of stalled region), thus the pressure gradient that retards the flow is $\Delta p / \mathrm{kg}$, 


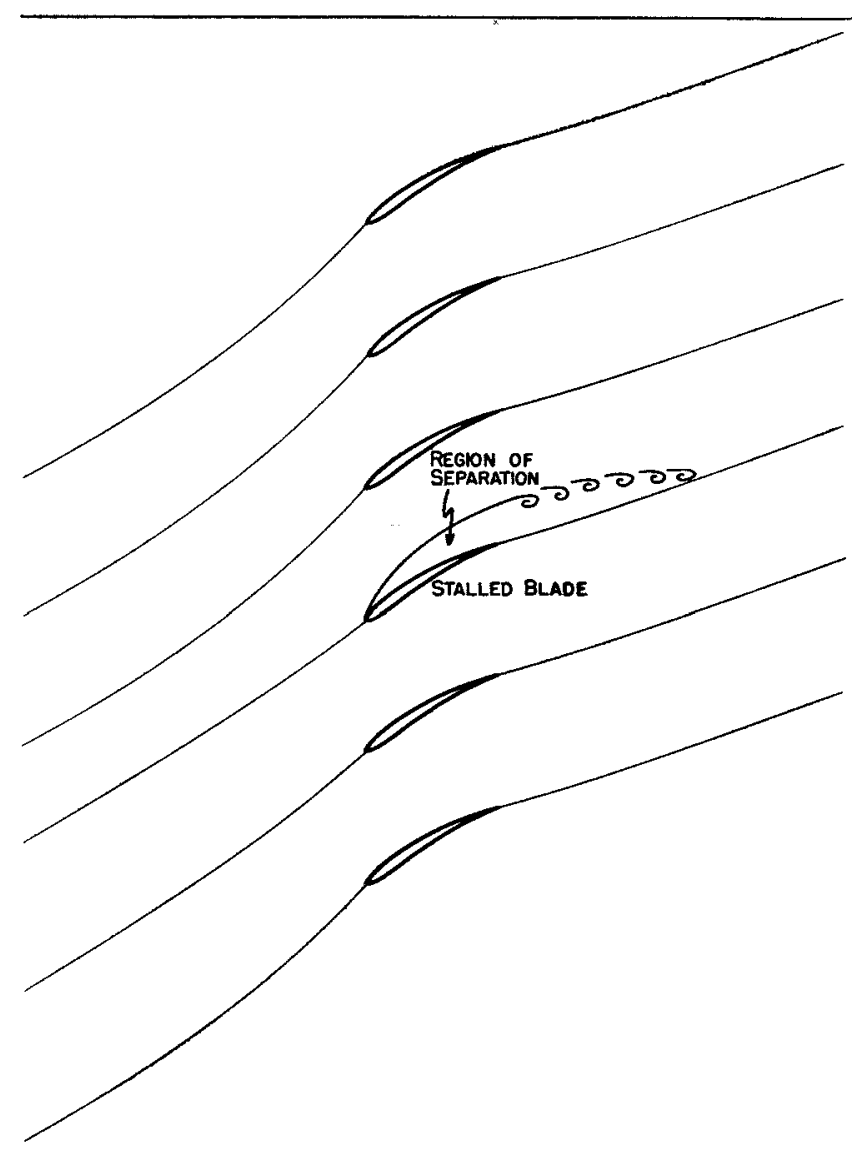

FrG. 1. Induced flow angles from stalled blade channel.

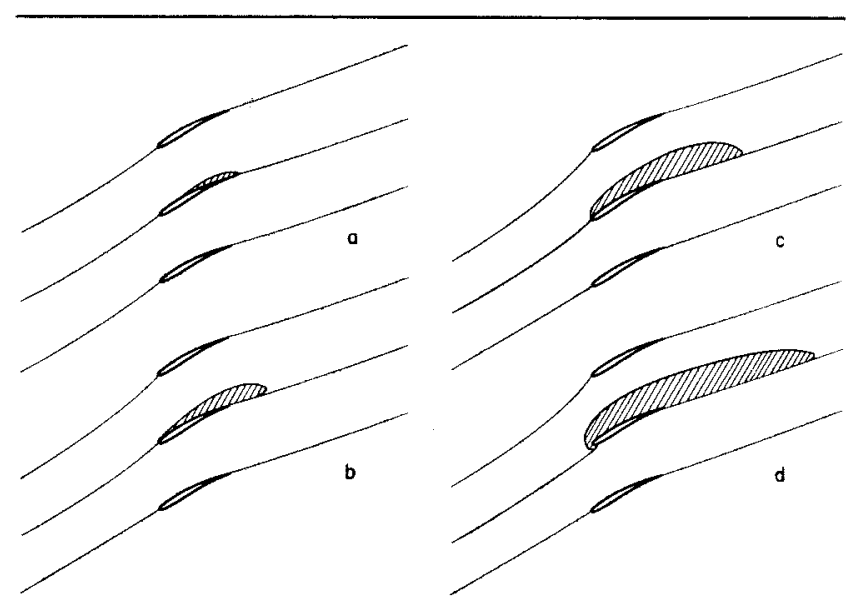

FrG. 2. Development of stalled region in a blade channel.

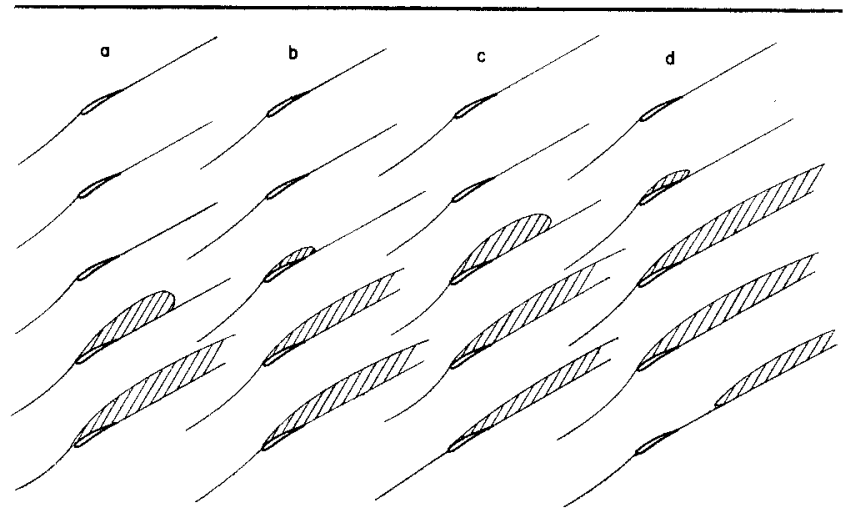

FIG. 3. Growth and distribution of stalled regions in blade row.

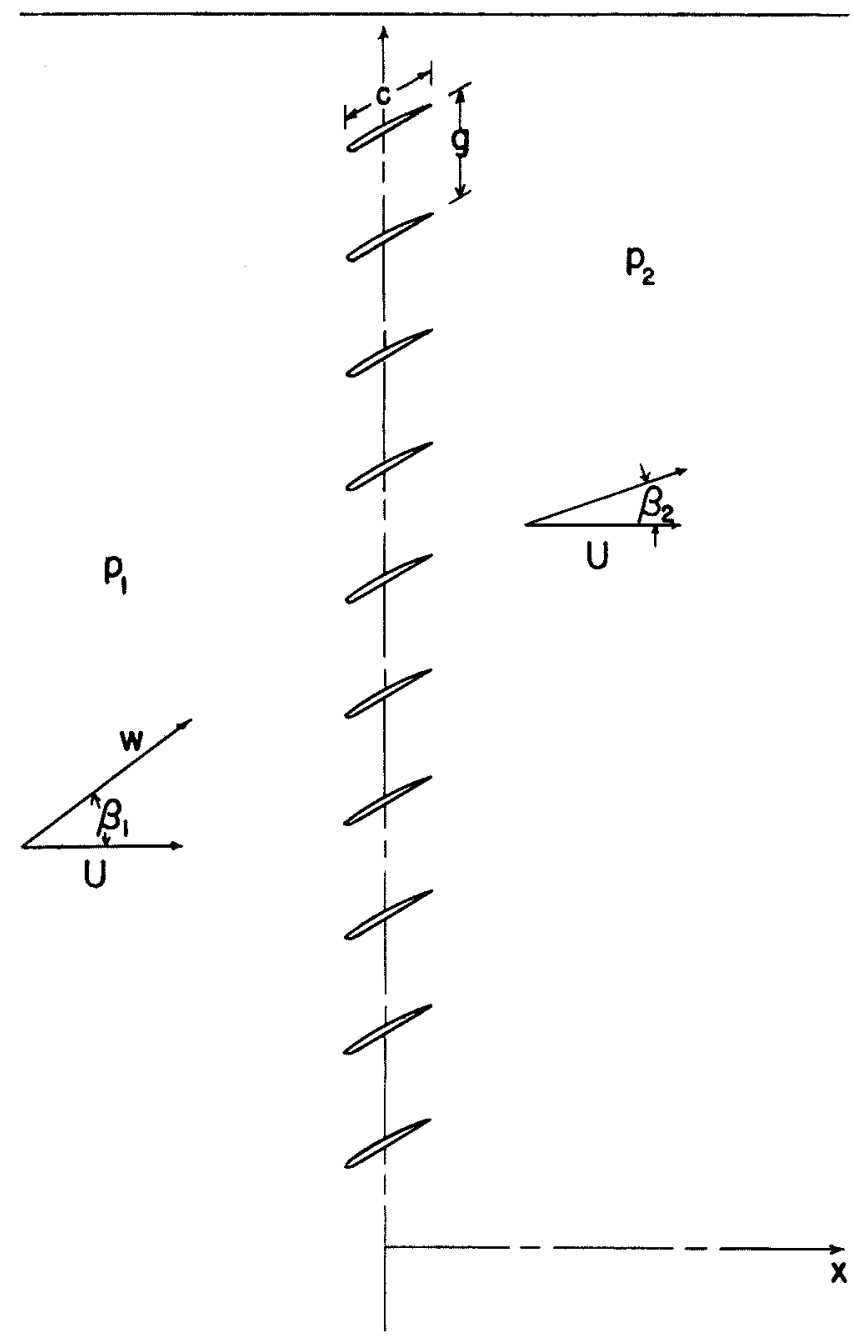

FIG. 4. Diagram of actuating line.

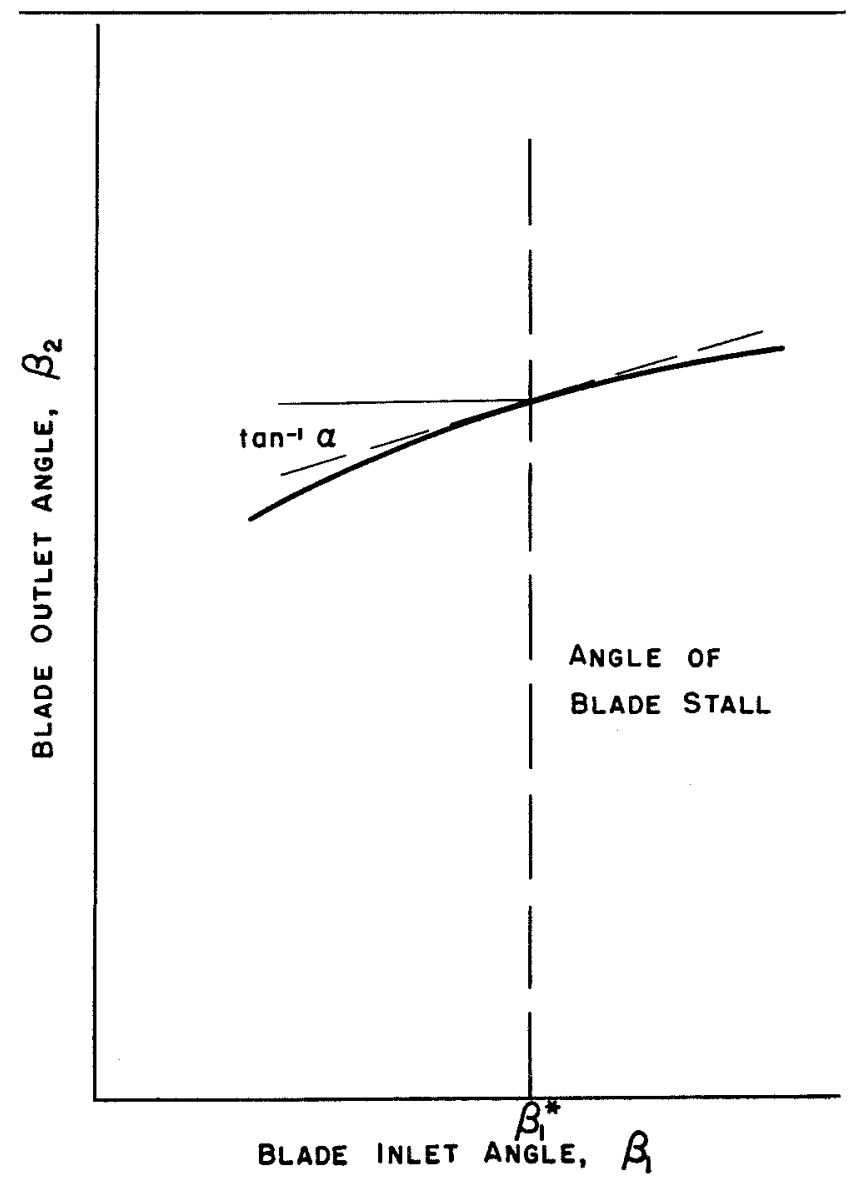

FIG. 5. Turning angle as function of inlet angle. 
where $\kappa$ is a constant of proportionality. At the initial instant of stall, where the approach velocity $w$ does not vary with distance upstream of the airfoil, the rate of change of $w$ is given by $\partial w / \partial t=-(1 / \rho)$ $(\Delta p / \kappa g)$. An estimate of the time $\tau$ required for the stalled pattern to develop may be obtained by assuming the transformation to continue at this rate. Now since there is no pressure rise through the blade row in stalled conditions, the flow approaching the blade row must undergo a change in velocity almost equal to $\Delta w$ in order that it may discharge to the pressure downstream of the blade row. Therefore, approximating $\partial w / \partial t$ by $\Delta w / \tau$, it follows that $\Delta w / \tau=$ $-(1 / \rho)(\Delta p / \kappa g)$ so that employing the relations between $\Delta p$ and $\Delta w$,

$$
w \tau / g=\kappa
$$

If the rotative speed of the compressor is $\Omega R$ and $m$ is the number of blades, then

$$
w \tau / g=[w /(\Omega R)][(\Omega R m \tau) /(m g)]
$$

where $w /(\Omega R)$ is usually about unity and for the present consideration is constant. But $m g=2 \pi R$ and the angular velocity of stall rotation is $\omega=2 \pi / m \tau$, since $m \tau$ is the time required to stall the total number of blades. Thus approximately $w \tau / g=\Omega / \omega$, the ratio of blade speed to that of stall propagation. Then since $w \tau / g=\kappa$ the ratio of stall propagation and rotor speeds is constant, $\omega / \Omega=1 / \kappa$, and in particular is independent of the blade gap as indicated in experiment.

It is usual that several blades are involved in observed stall propagation so that the blade spacing is not the significant dimension but rather the length of the stalled region. Consequently it is appropriate to simplify the model to an actuating line across which the velocities and pressure may change discontinuously. It is convenient to think of this simplification carried out by allowing the chord and gap to vanish simultaneously while their ratio remains fixed. Then it remains only to assign realistic conditions on the change of flow properties across the line.

To follow the convention found convenient in compressor practice, let the local inlet flow angle $\beta_{1}$ be the independent variable, Fig. 4 , in describing the characteristics of the cascade. The performance of the cascade is completely defined by prescribing (1) the local turning angle or the outlet flow angle and (2) the local static pressure rise across the cascade. The discharge angle $\beta_{2}$ is a well-determined function of the inlet angle, and, in the absence of losses, the static pressure rise is then fixed also. For considering the flow with respect to the blade row in question and accounting for the fact that the velocity $U$ normal to the cascade is equal ahead and behind the cascade, the Bernoulli relation gives simply $\Delta p=(1 / 2) \rho U^{2}$ $\left(\tan ^{2} \beta_{1}-\tan ^{2} \beta_{2}\right)$. By virtue of the dependence of $\beta_{2}$ upon $\beta_{1}$, the pressure rise across the cascade is determined. Now when a relation between $\Delta p$ and $\beta_{1}$ different from this is assigned, it can be possible only because the total pressure changes. Consequently choosing $\Delta p\left(\beta_{1}\right)$ is equivalent to assigning a value of the total pressure loss at each value of the inlet angle.

During stall propagation the entire blade row is operating sufficiently close to the angle of stall that the cascade characteristic need be approximated near this value; let $\beta_{1}{ }^{*}$ be the inlet flow angle for which the cascade stalls. Locally the turning angle is a linear function of the inlet angle as shown in Fig. 5, and there is experimental evidence to show that it is not profoundly influenced by the stall. Then it may be characterized by a discharge angle $\beta_{2}{ }^{*}$ and a slope of magnitude $a$ just prior to stall. The pressure rise may likewise be approximated by a linear function of the inlet angle below the stall angle, but it changes discontinuously at $\beta_{1}{ }^{*}$. According to the discussion of the stalling mechanism, the static pressure rise across the cascade may be approximated reasonably well by assuming it to vanish above the stall angle, to have a value $\Delta p^{*}$ at $\beta_{1}{ }^{*}$, and to have a slope $b$ just prior to stall. This approximation to the pressure rise characteristic is shown in Fig. 6 .

Such characterization of the cascade performance and stalling process differs from that employed by Emmons or by Sears in either of his analyses. ${ }^{4,5}$ The differences are not of great importance so far as the unstalled cascade characteristic is concerned, for any pair of performance parameters which depend upon the upstream flow angle is adequate, although not equally convenient. For example, the performance of a cascade may be expressed by giving the lift coefficient $C_{L}$ and the drag coefficient $C_{D}$ as functions of the angle of attack. Properly these coefficients are based upon the geometric mean of the upstream and downstream velocity vectors and the angle of attack measured with respect to its direction $\beta_{m}$. Then if $F_{x}$ and $F_{y}$ are the blade force components in the $x$ and $y$ directions, respectively, referring to Fig. 7 , the lift and drag coefficients are just

$$
\begin{gathered}
C_{L}=\left(-F_{x} \sin \beta_{m}+F_{y} \cos \beta_{m}\right) \div \\
{\left[(c / 2) \rho U^{2}\left(1+\tan ^{2} \beta_{m}\right)\right]} \\
C_{D}=\left(F_{x} \cos \beta_{m}+F_{y} \sin \beta_{m}\right) \div \\
{\left[(c / 2) \rho U^{2}\left(1+\tan ^{2} \beta_{m}\right)\right]}
\end{gathered}
$$

and the angle of attack is simply

$$
\beta_{m}-\alpha_{0} \equiv \tan ^{-1}\left[\left(\tan \beta_{1}+\tan \beta_{2}\right) / 2\right]
$$

where $\alpha_{0}$ is an arbitrary angle of zero lift. An elementary momentum calculation shows that $F_{y}=\rho U g(U$ $\tan \beta_{1}-U \tan \beta_{2}$ ), while the axial force results from pressure difference and is $F_{x}=-\Delta p g$, where $\Delta p$ is the static pressure rise across the blade row. Now if $C_{L}$ and $C_{D}$ are specified as functions of the angle of attack, then clearly the expressions for $F_{x}$ and $F_{y}$ given above determine $\beta_{2}$ and $\Delta p$ as functions of $\beta_{1}$. Therefore this viewpoint is equivalent to that used in the present analysis. Using the blade channel approach, the outlet angle $\beta_{2}$ and the total pressure loss $\Delta p_{t}$ are specified as functions of the inlet angle $\beta_{1}$. 


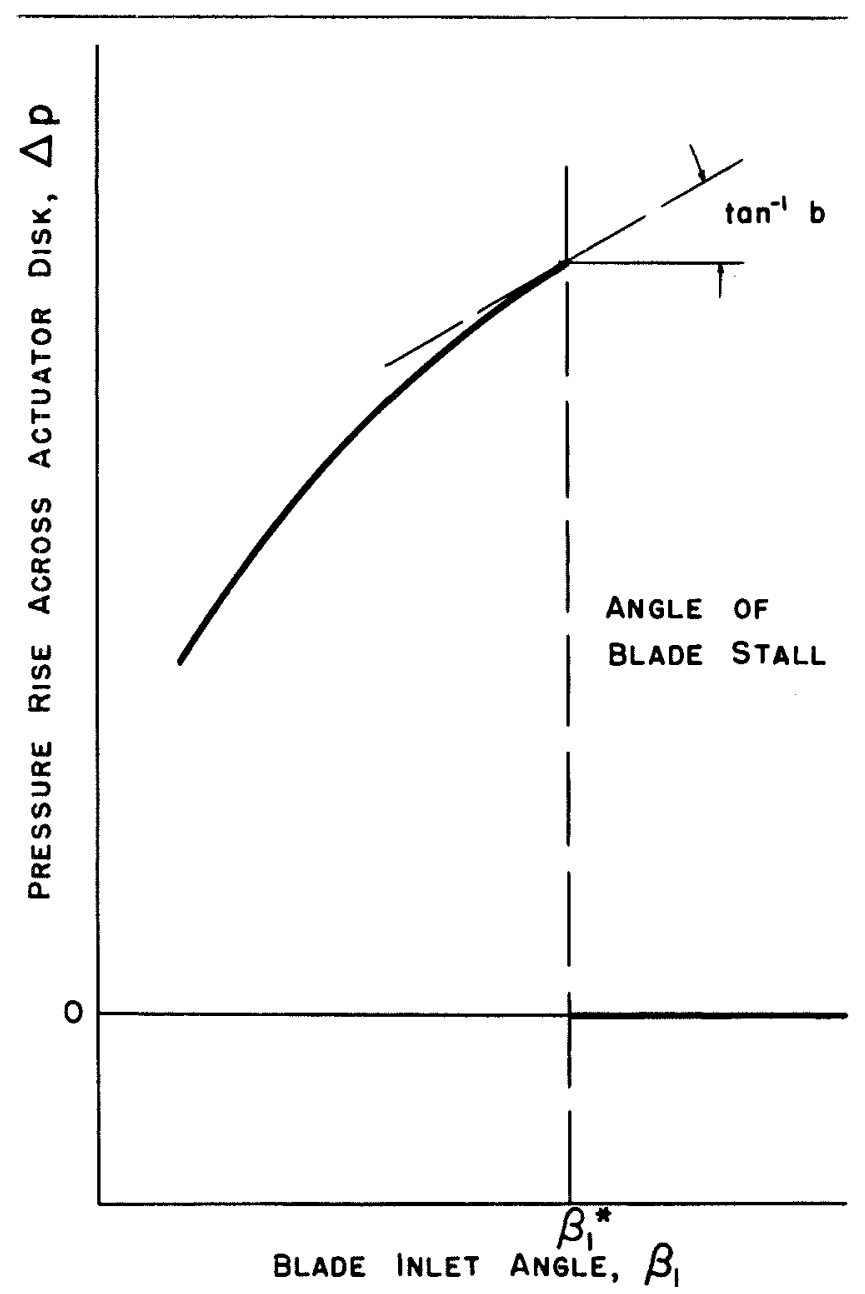

Fro. fi. Pressure rise as a function of inlet angle.

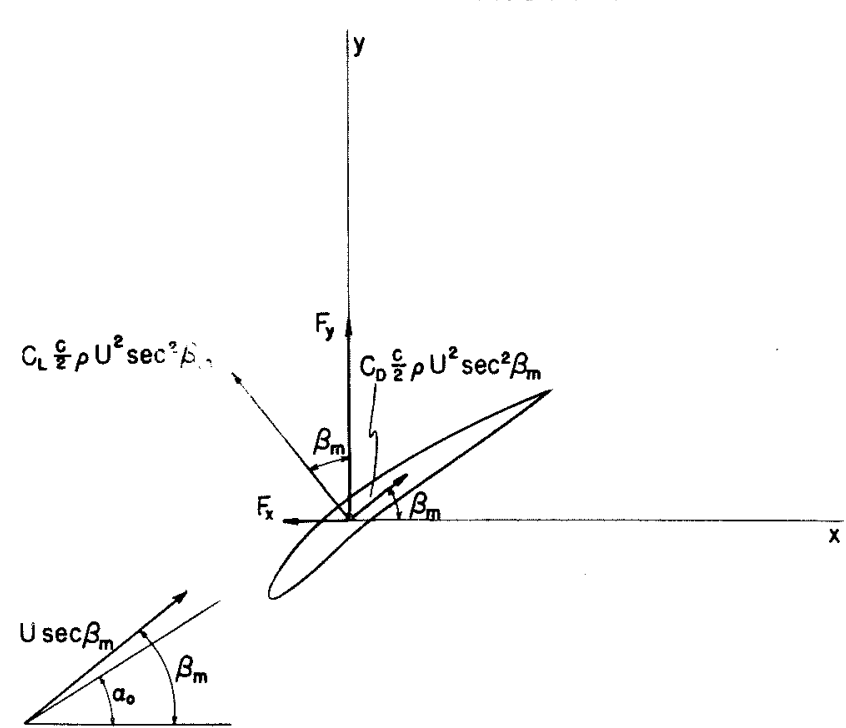

FIG. 7. Forces and velocities on cascade according to airfoil theory.

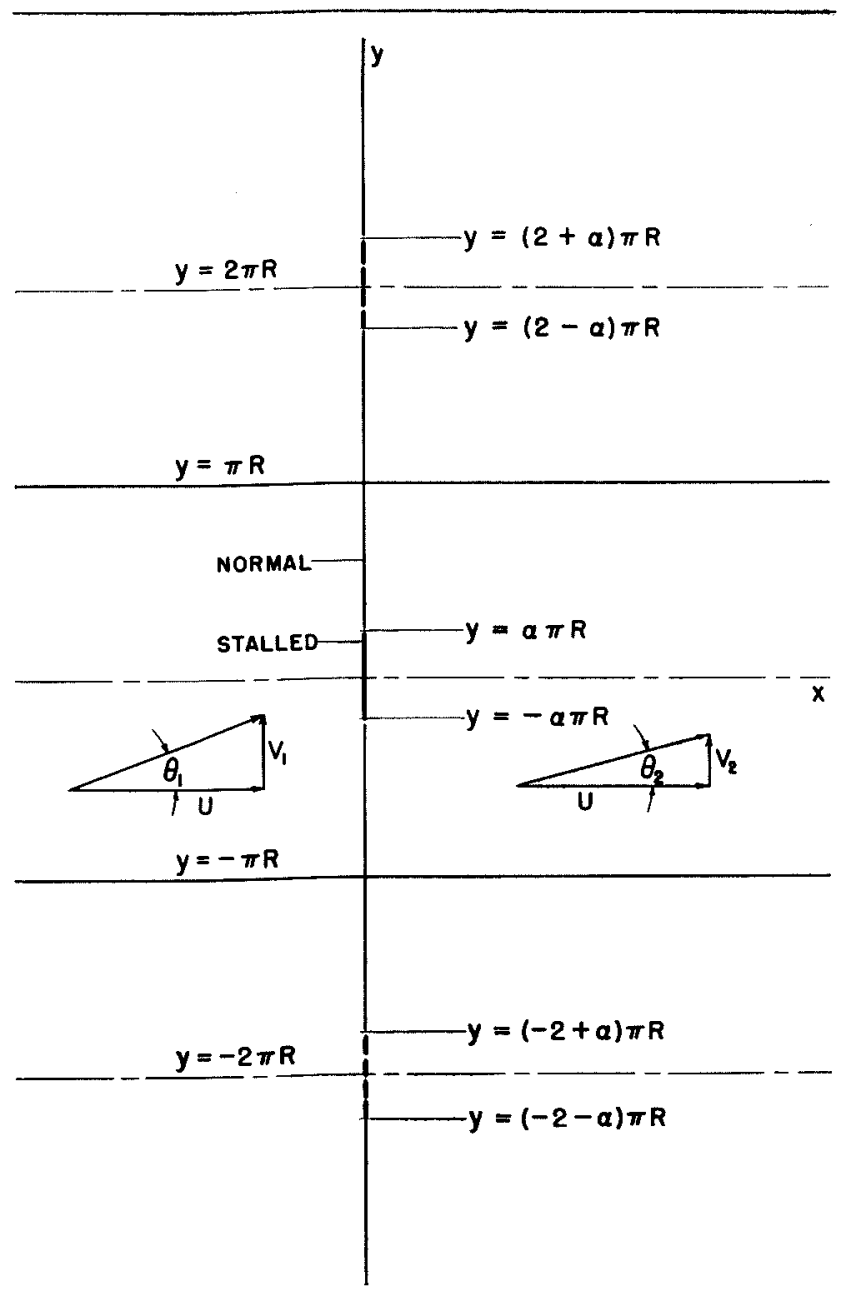

FIG. 8. Pattern of stalled and unstalled regions on actuating line.

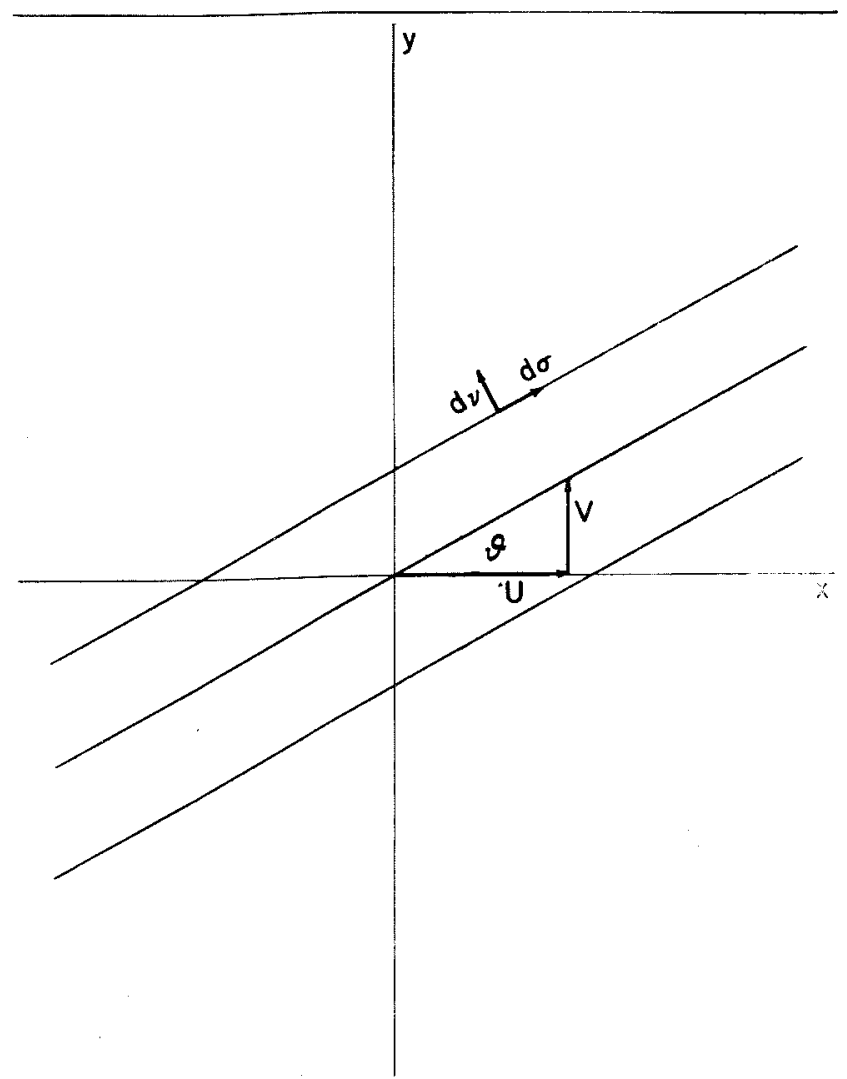

FIG. 9. Notation of coordinates and perturbations for flow along undisturbed streamlines. 
This is closely related to the specification employed in the present analysis, for clearly

$$
\Delta p=(1 / 2) \rho U^{2}\left(\tan ^{2} \beta_{1}-\tan ^{2} \beta_{2}\right)-\Delta p_{t}
$$

and hence $\Delta p\left(\beta_{1}\right)$ and $\beta_{2}\left(\beta_{1}\right)$ may be obtained directly.

It is interesting to compare the mechanisms of stall prescribed by Emmons ${ }^{1}$ and that suggested in the present analysis. Emmons utilizes the fact that stall restricts the flow through a stalled blade channel and recognizes that the development of steady stalled flow from the initial separated flow requires a time that is the governing factor in the rate of stall propagation. However this time is not calculated from the details of the nonsteady motion, and consequently no stall propagation speed may be determined. Emmons and his co-workers seemingly overlook the fact that the characteristic time is proportional to the peripheral extent of the stalled region and therefore draw the erroneous conclusion that stall propagation speed is proportional to the wave length. It must be recognized, however, that Emmons' mechanism contains elements that, if pursued to their logical conclusion, would produce propagation speeds essentially in agreement with those of the present analysis. The fact that Emmons et al. do not account for the influence of flow downstream of the blade row is a significant but not a decisive shortcoming. The "amplitude" of stall and hence its peripheral extent and associated pressure loss are essentially nonlinear effects that, because his mechanism is not employed to full advantage, Emmons does not determine.

In his initial treatment, Sears ${ }^{4}$ employs the approach of nonstationary airfoil theory, assuming an irregularity in lift near the stall angle but neglecting losses associated with stall. $\mathrm{He}$ assumes, moreover, that the actual stall lags a certain arbitrary phase behind the occurrence of critical inlet angle, this lag presumably being associated with the time required for boundary-layer separation, satisfying the Kutta condition or some other real fluid phenomena. It would seem that the most serious defect of this mechanism is its failure to account for the sharp increase in drag associated with stall. At the suggestion of Rannie, Sears improved his cascade characteristic in reference 5 to account for drag or pressure loss at the stall. He employed the conventional "channel theory" with the reasonable restriction that variation of discharge angle with inlet flow angle is neglected; the concept of stall phase lag, however, was retained. Since Sears solves the nonstationary flow problem for both cascade prescriptions, the velocity of stall propagation is determined except for influence of the arbitrary phase lag. Under special circumstances described later, the stall propagation speed computed under this channel theory agrees with the result developed herein. In both cases, however, Sears linearizes the cascade performance in the neighborhood of stall and consequently is unable to determine values for the extent of stall region or the pressure loss associated with stall.

\section{General Theory of Stall Propagation in Single} BLADE Rows

Consider a cascade formed by an annular element of radius $R$ from a rotating blade row. During normal operation the flow relative to the cascade is represented (Fig. 4) by a constant component $U$ of axial velocity and constant flow angles $\beta_{1}$ upstream of the cascade and $\beta_{2}$ downstream. Corresponding uniform values of static pressure $p_{1}{ }^{(0)}$ and $p_{2}{ }^{(0)}$ exist ahead of and behind the blade row, and, if $\beta_{1}$ is close to the stall angle $\beta_{1}{ }^{*}$, the pressures satisfy the relation

$$
p_{2}{ }^{(0)}-p_{1}{ }^{(0)} \equiv \Delta p=\Delta p^{*}+b\left(\beta_{1}-\beta_{1}{ }^{*}\right)(1 / 2) \rho U^{2}
$$

During stall propagation deviations from the uniform state occur which depend upon both location and time. The problem may be reduced to one of steady-state flow, however, by referring to a coordinate system $x, y$ moving with the velocity $\omega R$ of the stalled region. Let $V$ be the undisturbed tangential velocity component in this system; the quantities $u, v$ are the velocity perturbations and $p$ the pressure perturbation associated with stall propagation. A subscript 1 will denote conditions ahead of the blade row and a subscript 2 , those behind.

The correspondence between inlet and outlet flow angles necessitates that perturbations $\Delta \beta_{1}$ and $\Delta \beta_{2}$ of the inlet and outlet flow angles satisfy the relation

$$
\Delta \beta_{2}=a \Delta \beta_{1}
$$

so long as the inlet angle is near the stalling angle. In prescribing the static pressure jump across the cascade, it is necessary to distinguish between stalled and unstalled regions. Denoting the compressor circumference by $2 \pi R$ on the $y$ axis, the stall will be assumed to cover a fraction $\alpha$ of the circumference extending over the interval $-\alpha \pi \leq y / R \leq \alpha \pi$. This pattern is repeated with a period $2 \pi$ so that, as indicated in Fig. 8, the actuating line is stalled in the regions $(2 n-\alpha) \pi \leq$ $y / R \leq(2 n+\alpha) \pi$ and unstalled elsewhere. Therefore the perturbation pressures $p_{1}(0, y)$ and $p_{2}(0, y)$ immediately ahead of and behind the cascade, satisfy the condition

$$
\begin{aligned}
& \frac{p_{2}(0, y)-p_{1}(0, y)}{(1 / 2) \rho U^{2}}= \\
& \left\{\begin{array}{cc}
-\frac{\Delta p^{*}}{(1 / 2) \rho U^{2}}-b\left(\beta_{1}-\beta_{1}{ }^{*}\right) ;(2 n-\alpha) \pi \leq \frac{y}{R} \leq \\
b \Delta \beta_{1} & (2 n+\alpha) \pi \\
\text {; elsewhere }
\end{array}\right.
\end{aligned}
$$

Due to the fact that the undisturbed flow fields ahead of and behind the cascade are initially uniform, the pressure perturbations satisfy the Laplace equation

$$
\begin{aligned}
\left(\partial^{2} p_{1} / \partial x^{2}\right)+\left(\partial^{2} p_{1} / \partial y^{2}\right) & = \\
\left(\partial^{2} p_{2} / \partial x^{2}\right)+\left(\partial^{2} p_{2} / \partial y^{2}\right) & =0
\end{aligned}
$$

Furthermore, the pressure perturbation due to the stall must vanish far upstream and assume, at most, a constant value far downstream. Then for a pre- 
scribed state of steady operation the cascade performance given by Eqs. (1) and (2) determines completely the pressure field for the corresponding state of stall propagation. Now it is required further that the inlet flow angle exceed $\beta_{1}{ }^{*}$ in the stalled regions and be less than $\beta_{1}{ }^{*}$ in the unstalled regions. These conditions may be met only when the stall propagation speed $\omega R$ and the length $2 \alpha \pi R$ of the stalled region are properly chosen. Thus if there is a condition of stall propagation which corresponds to a given set of inlet conditions, the peripheral extent of the stall and the rate of stall propagation are determined.

The flow angle deviations that are involved in the boundary values of the problem must be found from the pressure field. When the undisturbed flow angle is $\vartheta=\tan ^{-1}(V / U)$, then the disturbed flow angle $\vartheta+$ $\Delta \vartheta$ is related to the axial and tangential velocity perturbations as

$$
\tan (\vartheta+\Delta \vartheta)=(V+v) /(U+u)
$$

and since $u$ and $v$ are small

$$
\left(1+\tan ^{2} \vartheta\right) \Delta \vartheta=(v / u)-(u / U) \tan \vartheta
$$

These velocity disturbances are related to the pressure distribution through the equations of motion which, for the present purpose, are most conveniently written along and normal to the streamlines of the undisturbed flow. Let the coordinate along the streamline be $\sigma$ and that normal to the streamline, $\nu$, as indicated in Fig. 9. The equation of equilibrium along the streamline is

$$
\rho U(\partial / \partial \sigma)(u+v \tan \vartheta)=-\partial p / \partial \sigma
$$

and has an obvious integral which is the familiar linearized Bernoulli relation. The equilibrium equation normal to the streamline is

$$
\rho U(\partial / \partial \sigma)(v-u \tan \vartheta)=-\partial p / \partial \nu
$$

and, by reference to $\mathrm{Eq}$. (4), is clearly related to the angle deviation $\Delta \vartheta$. In fact, Eq. (6) is just

$$
\rho U^{2}\left(1+\tan ^{2} \vartheta\right)(\partial / \partial \sigma)(\Delta \vartheta)=-\partial p / \partial \nu
$$

Furthermore, the continuity relation for this coordinate system says simply that

$$
(\partial / \partial \sigma)(u+v \tan \vartheta)=-(\partial / \partial \nu)(v-u \tan \vartheta)
$$

and consequently Eq. (5) reads

$$
\rho U^{2}\left(1+\tan ^{2} \vartheta\right)(\partial / \partial \nu)(\Delta \vartheta)=\partial p / \partial \sigma
$$

Then two dependent variables may be defined

$$
\begin{aligned}
P & =p /\left[(1 / 2) \rho U^{2}\right] \\
\theta & =2\left(1+\tan ^{2} \vartheta\right) \Delta \vartheta
\end{aligned}
$$

which reduce the equations of motion to

$$
\begin{gathered}
\partial P / \partial \sigma=\partial \theta / \partial \nu \\
\partial P / \partial \nu=-\partial \theta / \partial \sigma
\end{gathered}
$$

These are the familiar Cauchy-Riemann relations which state not only that $\theta$ (and hence $\Delta v$ ) is an harmonic function but that it is indeed the harmonic conjugate of $P$ and, further, that this useful relationship holds so long as the vorticity in the field is small.

Far upstream of the cascade both pressure and angle perturbations vanish and hence Eq. (5) may be integrated directly to

$$
2\left[\left(u_{1} / U\right)+\left(v_{1} / U\right) \tan \vartheta_{1}\right]=-P_{1}
$$

Noting that

$$
\partial p_{1} / \partial \nu_{1}=-(1 / 2) \rho U^{2}\left(\partial \theta_{1} / \partial \sigma\right)
$$

Eq. (6) also integrates in a similar manner to

$$
2\left[\left(v_{1} / U\right)-\left(u_{1} / U\right) \tan \vartheta_{1}\right]=\theta
$$

The local flow velocities $u_{1}$ and $v_{1}$ are then simply

$$
\begin{aligned}
2\left(1+\tan ^{2} \vartheta_{1}\right)\left(u_{1} / U\right) & =-P_{1}-\theta_{1} \tan \vartheta_{1} \\
2\left(1+\tan ^{2} \vartheta_{1}\right)\left(v_{1} / U\right) & =-P_{1} \tan \vartheta_{1}+\theta_{1}
\end{aligned}
$$

In order to reduce the problem to one of steady motion, the reference frame has been taken fixed in the rotating stall pattern, but when dealing with inlet and outlet angles of the blades it is necessary to consider angles that are measured with respect to the blades themselves. Since the variation in flow angle with respect to the blade is just

$$
\left(1+\tan ^{2} \beta\right) \Delta \beta=(v / U)-(u / U) \tan \beta
$$

it is convenient to write in general

$$
\begin{aligned}
& \left(1+\tan ^{2} \beta\right) \Delta \beta= \\
& \left(1+\tan ^{2} \vartheta\right) \Delta \vartheta-(\omega R / U)(u / U)
\end{aligned}
$$

where account has been taken of the fact that

$$
U \tan \beta-U \tan \vartheta=\omega R
$$

where $\omega$ is the angular velocity of stall propagation. In particular, the variation of inlet angle to the blade row is, using Eq. (10),

$2 \sec \beta_{1} \sec \vartheta_{1} \Delta \beta_{1}=P_{1}(0) \sin \left(\beta_{1}-\vartheta_{1}\right)+$

$$
\theta_{1}(0) \cos \left(\beta_{1}-\vartheta_{1}\right)
$$

Now the condition for the pressure jump given by Eq. (2) may be written explicitly as

$$
\left.\begin{array}{c}
P_{2}(0)-P_{1}(0)= \\
-\left[\frac{\Delta p^{*}}{(1 / 2) \rho U^{2}}+b\left(\beta_{1}-\beta_{1}^{*}\right)\right] ; \\
(2 n-\alpha) \pi \leq y / R \leq(2 n+\alpha) \pi \\
\frac{b}{2} \cos ^{2} \beta_{1}\left[\left(1+\frac{\omega R}{U} \sin \vartheta_{1} \cos \vartheta_{1}\right) \theta_{1}(0)+\right. \\
\left.\frac{\omega R}{U} \cos ^{2} \vartheta_{1} P_{1}(0)\right] ; \text { elsewhere }
\end{array}\right\}
$$

Downstream of the cascade the flow may be treated in a similar manner. In addition to satisfying the above condition on pressure jump, the discharge angle variation $\Delta \beta_{2}$ must bear the relation to the inlet angle prescribed by the cascade characteristic, Eq. (1). 
Then utilizing Eq. (12) and the fact that the axial velocity is continuous across the cascade, Eq. (1) may be written

$$
\begin{gathered}
\cos ^{2} \beta_{2}\left\{\theta_{2}(0)+(\omega R / U) \cos ^{2} \vartheta_{1}\left[P_{1}(0)+\theta_{1}(0) \tan \vartheta_{1}\right]\right\} \\
=a \cos ^{2} \beta_{1}\left\{\theta_{1}(0)+(\omega R / U) \cos ^{2} \vartheta_{1}\left[P_{1}(0)+\right.\right. \\
\left.\left.\theta_{1}(0) \tan \vartheta_{1}\right]\right\}
\end{gathered}
$$

Summarizing the problem, the pressure and angle perturbations vanish far upstream of the blade tow while the pressure perturbation $p_{2}(\infty)$ and the angle perturbation $\Delta \vartheta_{2}(\infty)$ may at most have constant values far downstream. Taken together with Eqs. (14) and (15), which respectively express the physical conditions that the pressure jump and the cascade discharge angle are those prescribed by the cascade performance, the problem is completely specified.

\section{Stall Propagation with Simple Cascade Characteristics}

The details of stall propagation are particularly simple when the cascade discharge angle is unaffected by the inlet angle and when the pressure rise across the cascade is zero or $\Delta p^{*}$ accordingly as the region in question is stalled or tunstalled. In high solidity cascades this condition may be nearly realized; in the present formulation it corresponds to the case when $a=b=0$. Then the pressure jump condition is simply

$$
\begin{aligned}
& P_{2}(0)-P_{1}(0)= \\
& \left\{\begin{array}{c}
-\frac{\Delta p^{*}}{(1 / 2) \rho U^{2}} ;(2 n-\alpha) \pi \leq \frac{y}{R} \leq(2 n+\alpha) \pi \\
0 \quad ; \text { elsewhere }
\end{array}\right\}
\end{aligned}
$$

Since $P_{1}$ and $P_{2}$ are both harmonic functions, it is convenient to commence construction of the solution by obtaining the harmonic function that assumes the values $P_{2}(0)-P_{1}(0)$ along the $y$-axis, is regular elsewhere, and approaches at most finite constant values far ahead and far behind the blade row. This is easily done by recalling the ideal flow field associated with an infinite row of sources or sinks. The complex potential of a unit source at the origin is just $[1 /(2 \pi)] \log z$ where $z=x+i y$ is an arbitrary point in the plane. The imaginary part of this general potential, the stream function, represents the flux of fluid across a curve connecting the point $z$ with any fixed point of the plane. If $z$ moves upward along the $y$-axis at small negative values of $x$, the flux increases by half the source strength as $z$ passes the origin, the change being discontinuous for sufficiently small values of $x$; and thus $\operatorname{Im}(1 / \pi) \log z$ possesses a unit jump at the origin. Then clearly the desired harmonic function may be constructed by a superposition of such jumps; more precisely it may be constructed by superposing a negative unit jump at each point $y=i(2 n-\alpha) \pi$ and a positive unit jump at each point $y=i(2 n+\alpha) \pi$. The positive jumps, considered separately, correspond to an infinite vertical row of sources with spacing $2 \pi R$, and the resulting complex potential is known (see, for example, Lamb') to be

$$
(1 / \pi) \log \{\sinh (1 / 2)[(z / R)+i \alpha \pi]\}
$$

The potential representing the negative unit jumps is entirely similar so that the desired harmonic function is

$$
\begin{aligned}
F(x, y)= & \frac{1}{\pi} \operatorname{Im} \log \left\{\frac{\sinh (1 / 2)[(z / R)-i \alpha \pi]}{\sinh (1 / 2)[(z / R)+i \alpha \pi]}\right\}= \\
& \frac{1}{\pi}\left(\tan ^{-1}\left\{\frac{\tan (1 / 2)[(y / R)-\alpha \pi]}{\tanh (1 / 2)(x / R)}\right\}-\right. \\
& \left.\tan ^{-1}\left\{\frac{\tan (1 / 2)[(y / R)+\alpha \pi]}{\tanh (1 / 2)(x / R)}\right\}\right)
\end{aligned}
$$

The function $F(x, y)$ is odd in $x$; in fact

$$
F\left(0^{-}, y\right)=\left\{\begin{array}{l}
1 \\
0
\end{array}\right\}
$$

while

$$
F\left(0^{+}, y\right)=\left\{\begin{array}{r}
-1 \\
0
\end{array}\right\}
$$

Furthermore, as $x \rightarrow \infty$,

$$
\begin{aligned}
& F(\infty, y)=\frac{1}{\pi}\{(1 / 2)[(y / R)-\alpha \pi]- \\
& (1 / 2)[(y / R)+\alpha \pi]\}=-\alpha
\end{aligned}
$$

and similarly

$$
F(-\infty, y)=\alpha
$$

Now the perturbation pressures $P_{1}$ and $P_{2}$ can differ from a multiple of $F$ only by an harmonic function that is regular except at certain points of the cascade. Its form is determined by the remaining condition to be imposed, Eq. (15), on the cascade discharge angle. From the fact that $P$ and $\theta$ are harmonic conjugates, it is clear that the supplementary harmonic function being sought can differ from the conjugate of $F$ by, at most, a constant. Denote this function $G(x, y)$ such that

$$
\partial G / \partial x=\partial F / \partial y, \partial G / \partial y=-\partial F / \partial x
$$

Then the pressure field may be written

$$
\left.\begin{array}{rl}
P_{1}=A[F(x, y)-\alpha]+B G(x, y) \\
P_{2}=\left[\frac{\Delta p^{*}}{(1 / 2) \rho U^{2}}-A\right][F(x, y)+\alpha]+ \\
B G(x, y)-\alpha \frac{\Delta p^{*}}{(1 / 2) \rho U^{2}}
\end{array}\right\}
$$

and the field of flow angle perturbations as

$$
\left.\begin{array}{r}
\theta_{1}=-A G(x, y)+B[F(x, y)-\alpha] \\
\theta_{2}=-\left[\frac{\Delta p^{*}}{(1 / 2) \rho U^{2}}-A\right] G(x, y)+ \\
B[F(x, y)+\alpha]+\alpha C
\end{array}\right\}
$$

where $A, B$, and $C$ are constants to be determined.

Now the angular velocity of stall propagation $\omega$ and the fraction $\alpha$ of the compressor circumference which 
is stalled for a given inlet angle are determined by the perturbation of inlet angle which is induced by the stall itself. Referring to Eq. (13) and substituting the values for $P_{1}$ and $\theta_{1}$, the inlet angle perturbation may be written

$2 \sec \beta_{1} \sec \vartheta_{1} \Delta \beta_{1}=\left[-A \cos \left(\beta_{1}-\vartheta_{1}\right)+\right.$

$$
\left.B \sin \left(\beta_{1}-\vartheta_{1}\right)\right] G(0, y)+
$$

$\left[A \sin \left(\beta_{1}-\vartheta_{1}\right)+B \cos \left(\beta_{1}-\vartheta_{1}\right)\right]\left[F\left(0^{-}, y\right)-\alpha\right]$

But since

$$
G(x, y)=\frac{1}{\pi} R l \log \left\{\frac{\sin (1 / 2)[(z / R)-i \alpha \pi]}{\sin (1 / 2)[(z / R)+i \alpha \pi]}\right\}
$$

it is clear that $G(0, y)$ and hence, in general, $\Delta \beta_{1}(0, y)$ have logarithmic singularities at the edges of the stalled region, $y / R= \pm \alpha \pi$. Therefore, the only possibility of satisfying the condition that $\beta_{1}=\beta_{1}{ }^{*}$ at these points is that the coefficient of $G(0, y)$ vanish in the expression for the inlet angle perturbation. Therefore

$$
A \cos \left(\beta_{1}-\vartheta_{1}\right)=B \sin \left(\beta_{1}-\vartheta_{1}\right)
$$

or

$$
A\left(1+\tan \beta_{1} \tan \vartheta_{1}\right)=B\left(\tan \beta_{1}-\tan \vartheta_{1}\right)
$$

and employing this to simplify Eq. (20), the expression for the inlet angle perturbation becomes simply

$$
\begin{aligned}
& \Delta \beta_{1}(0, y)=\frac{A\left[F\left(0^{-}, y\right)-\alpha\right]}{2\left(\tan \beta_{1}-\tan \vartheta_{1}\right)}= \\
& \frac{A}{2(\omega R / U)}\left[F\left(0^{-}, y\right)-\alpha\right]
\end{aligned}
$$

The determination of constants $A$ and $B$ requires imposing the condition on cascade discharge angle given by $\mathrm{Eq}$. (15). In the present example where $a=0, \Delta \beta_{2}=0$ and consequently

$\theta_{2}(0)+(\omega R / U) \cos ^{2} \vartheta_{1}\left[P_{1}(0)+\theta_{1}(0) \tan \vartheta_{1}\right]=0$

Substitution of the proposed solutions [Eqs. (18) and (19) J gives, due to the linear independence of $F\left(0^{-}, y\right)$ and $G(0, y)$

$$
\begin{gathered}
{[(\omega R / U) A]-\left[2\left(1-\tan ^{2} \vartheta_{1}\right)-\right.} \\
\left.\left(1+\tan \beta_{1} \tan \vartheta_{1}\right)\right] B=0 \\
{\left[2\left(1+\tan ^{2} \vartheta_{1}\right)-\left(1+\tan \beta_{1} \tan \vartheta_{1}\right)\right] A+} \\
{[(\omega R / U) B]=\left\{\Delta p^{*} /\left[(1 / 2) \rho U^{2}\right]\right\}}
\end{gathered}
$$$$
\left(1+\tan ^{2} \vartheta_{1}\right)
$$

$\left(1+\tan ^{2} \vartheta_{1}\right) C=[(\omega R / U) A]-$

$$
\left[2\left(1+\tan ^{2} \vartheta_{1}\right)-\left(1+\tan \beta_{1} \tan \vartheta_{1}\right)\right]
$$

It appears immediately from Eq. (26) that the downstream flow angle $\Delta \vartheta_{2}(\infty)$ is unperturbed by the stall. Comparison of Eqs. (21) and (25) gives directly that

$$
A=(1 / 2)\left\{\Delta p^{*} /\left[(1 / 2) \rho U^{2}\right]\right\}
$$

There remain two homogeneous relations, Eqs. (21) and (24), to be satisfied and this may be accomplished only if their coefficients are related-that is, if there exists a relation between the inlet angle and the speed of stall propagation. Dividing these two relations gives simply

$$
(\omega R / U)^{2}=1+\tan ^{2} \vartheta_{1}
$$

or in terms of the inlet angle relative to the blades,

$\omega R / U=(1 / 2)\left[\left(1+\tan ^{2} \beta_{1}\right) / \tan \beta_{1}\right]=\csc 2 \beta_{1}$

As indicated while discussing the physical mechanism of stall, the propagation speed is independent of the peripheral extent of the stalled region in conformity with the experiments. Since the critical item in determining the propagation speed is the inlet angle to the cascade, it seems reasonable that the propagation speed is independent of the stream deflection in passing through the blade row. It is not surprising that this is identical with the result obtained by Sears, ${ }^{5}$ when the phase lag associated with his assumed pressure loss vanishes.

The extent of the stalled region must be determined through consideration of the actual distribution of inlet flow angle which is now known explicitly to be

$$
\begin{aligned}
\Delta \beta_{1}=\{1 /[4(\omega R / U)]\}\left[F\left(0^{-}, y\right)-\alpha\right] \\
\left\{\Delta p^{*} /\left[(1 / 2) \rho U^{2}\right]\right\}
\end{aligned}
$$

The total angle of attack $\beta_{1}+\Delta \beta_{1}$ must equal $\beta_{1}{ }^{*}$ at the edges of the stalled region, and, since $F\left(0^{-}, y\right)$ is discontinuous at these points, the proper limiting value is its mean, which is equal to $1 / 2$. Therefore the condition $\Delta \beta_{1}(0, \pm \alpha \pi R)=\beta_{1}{ }^{*}-\beta_{1}$ determines the fraction of stalled blade region to be

$\alpha=(1 / 2)-\left(\beta_{1}{ }^{*}-\beta_{1}\right)\left(\csc 2 \beta_{1}\right) 4\left\{\left[(1 / 2) \rho U^{2}\right] / \Delta p^{*}\right\}$

So far as the dependence of $\alpha$ upon $\beta_{1}$ is concerned, it is predominantly the factor $\beta_{1}{ }^{*}-\beta_{1}$ which affects the result, the variation of $\csc 2 \beta_{1}$ being small under usual circumstances. It is clear that the stall will first appear for angles below that which would cause the cascade to stall uniformly. On the other hand, the inlet angle $\beta_{1}$ must exceed $\beta_{1}{ }^{*}$ by a certain amount before the entire blade row is stalled. It must be kept in mind, however, that the solution becomes inaccurate long before this condition is reached since the linearization rests on the assumption that $\alpha \ll 1$.

The propagation speed $\omega R / U$ is shown in Fig. 10 as a function of the inlet angle. It is of interest to note that the propagation speed is particularly insensitive to variations of inlet flow angle in the neighborhood of $\beta_{1}=\pi / 4$, a value that is usual for normal axial compressors. This is certainly related to the observed fact that the propagation speed does not vary markedly as the compressor is throttled. The manner in which the stalled fraction $\alpha$ increases with inlet angle is shown in Fig. 11 for the particular values of $\beta_{1}{ }^{*}=\pi / 4$ and $\Delta p^{*} /\left[(1 / 2) \rho U^{2}\right]=0.5$. It appears that a rather narrow range of inlet angle is involved in passing from the first appearance of stall propagation to a completely stalled blade row. While this is probably realistic near the onset of stall, it is certainly in error as the stalled region grows. The pressure rise $\Delta p^{*}$ has 
a strong influence upon the angle at which stall first appears as well as the range of angles over which stall propagation is possible. The angle $\beta_{1}$ for incipient stall propagation is decreased for a heavily loaded blade row. Physically this says simply that the "relief" on other blades due to a region of stalled blades is greater when the stall involves a larger loss of pressure. To illustrate this influence, the variation of $\alpha$ with $\beta_{1}$ is shown in Fig. 12 for values of $\Delta p^{*} /\left[(1 / 2) \rho U^{2}\right]=0.5$, 1.0 , and 2.0 with the inlet angle $\beta_{1}{ }^{*}$ at which stall occurs equal to $45^{\circ}$.

\section{Stall Propagation with General Cascade Characteristics}

The results of the previous section are somewhat modified when the pressure rise across the cascade and the discharge angle from the cascade depend upon the inlet angle near the stall. The pressure field ahead of the blade row is of the same form as before

$$
P_{1}(x, y)=A[F(x, y)-\alpha]+B G(x, y)
$$

but the downstream pressure field must be modified in order to satisfy the general condition on pressure jump given by Eq. (2). In the stalled region the pressure drop is now $\left\{\Delta p^{*} /\left[(1 / 2) \rho U^{2}\right]\right\}+b\left(\beta_{1}-\beta_{1}^{*}\right)$ so that the pressure field downstream of the cascade could be chosen as

$$
\begin{array}{r}
P_{2}(x, y)=\left(\left\{\Delta p^{*} /\left[(1 / 2) \rho U^{2}\right]\right\}+b\left(\beta_{1}-\beta_{1}{ }^{*}\right)-A\right) \times \\
{[F(x, y)+\alpha]+B G(x, y)-} \\
\alpha\left(\left\{\Delta p^{*} /\left[(1 / 2) \rho U^{2}\right]\right\}+b\left(\beta_{1}-\beta_{1}{ }^{*}\right)\right)
\end{array}
$$

were it not for the fact that the pressure rise across the cascade is proportional to the inlet angle perturbation in the installed region. Now since the formal expression for the upstream pressure field is identical with that employed in the solution for simple cascade characteristics, the expression for $\Delta \beta_{1}$ is general and hence

$$
P_{2}(0, y)-P_{1}(0, y)=(b / 2)[A /(\omega R / U)](1-\alpha)
$$

in the unstalled region. The appropriate expression for $P_{2}(x, y)$ is therefore

$$
\begin{aligned}
& P_{2}(x, y)=\left[\frac{\Delta p^{*}}{(1 / 2) \rho U^{2}}+b\left(\beta_{1}-\beta_{1}^{*}\right)-A\right] \times \\
& {[F(x, y)+\alpha]+B G(x, y)-\frac{b}{2} \frac{A}{(\omega R / U)}(1-\alpha) \times} \\
& {[F(x, y)+1]-\alpha\left[\frac{\Delta p^{*}}{(1 / 2) \rho U^{2}}+b\left(\beta_{1}-\beta_{1}^{*}\right)\right]}
\end{aligned}
$$

inasmuch as $F\left(0^{+}, y\right)+1$ vanishes in the stalled region and is equal to unity elsewhere. The corresponding flow angle variations are then

$$
\begin{gathered}
\theta_{1}=-A G(x, y)+B[F(x, y)-\alpha] \\
\theta_{2}=-\left[\frac{\Delta p^{*}}{(1 / 2) \rho U^{2}}+b\left(\beta_{1}-\beta_{1}^{*}\right)-A\right] G(x, y)+ \\
B[F(x, y)+\alpha]+\frac{b}{2} \frac{A}{(\omega R / U)}(1-\alpha) G(x, y)+\alpha C
\end{gathered}
$$

where, as before, $C$ is a constant to be determined.
The condition on the outlet angle, given by Eq. (15), now gives, upon substitution of the explicit forms for the pressure and angle perturbation fields, three relations to determine the constants $A, B$, and $C$.

$$
\begin{gathered}
\left(\tan \beta_{1}-\tan \vartheta_{1}\right)\left[1-a\left(\cos ^{2} \beta_{1} / \cos ^{2} \beta_{2}\right)\right] A- \\
\left\{2\left(1+\tan ^{2} \vartheta_{1}\right)-\left[1-a\left(\cos ^{2} \beta_{1} / \cos ^{2} \beta_{2}\right)\right]\right. \\
\left.\left(1+\tan \vartheta_{1} \tan \beta_{1}\right)\right\} B=0 \\
\left\{\left[2+\frac{b}{2} \frac{(1-\alpha)}{(\omega R / U)}\right]\left(1+\tan ^{2} \vartheta_{1}\right)-\left(1-a \frac{\cos ^{2} \beta_{1}}{\cos ^{2} \beta_{2}}\right) \times\right. \\
\left.\left(1+\tan \vartheta_{1} \tan \beta_{1}\right)\right\} A+\left(\tan \beta_{1}-\tan \vartheta_{1}\right) \times \\
\left(1-a \frac{\cos ^{2} \beta_{1}}{\cos ^{2} \beta_{2}}\right) B=\left(1+\tan ^{2} \vartheta_{1}\right)\left[\frac{\Delta p^{*}}{(1 / 2) \rho U^{2}}+\right. \\
\left.\quad b\left(\beta_{1}-\beta_{1}{ }^{*}\right)\right] \\
C=\left(\tan \beta_{1}-\tan \vartheta_{1}\right)\left[1-a\left(\cos ^{2} \beta_{1} / \cos ^{2} \beta_{2}\right)\right] A- \\
\left\{2\left(1-\tan ^{2} \vartheta_{1}\right)-\left[1-a\left(\cos ^{2} \beta_{1} / \cos ^{2} \beta_{2}\right)\right]\right. \\
\left.\left(1+\tan \vartheta_{1} \tan \beta_{1}\right)\right\} B \equiv 0
\end{gathered}
$$

where as before the constant $C=0$ so that the flow angle far downstream is unperturbed by the stall. Furthermore, it follows by comparison of Eq. (21) with Eq. (36) that the constant $A$ is just

$$
\begin{aligned}
A=\frac{1}{2}\left\{\left[\frac{\Delta p^{*}}{(1 / 2) \rho U^{2}}+b\left(\beta_{1}-\beta_{1}{ }^{*}\right)\right] \div\right. \\
\\
{\left[1+\frac{b}{4} \frac{(1-\alpha)}{(\omega R / U)}\right] }
\end{aligned}
$$

which reduces to the previous result [Eq. (27)] when $b=0$. Now by dividing Eqs. (35) and (21), as was done for the simple cascade characteristic, the corresponding result is obtained for the speed of stall propagation

$$
(\omega R / U)^{2}=1+\tan ^{2} \vartheta_{1}+
$$

$$
\left[2 a \cos ^{2} \beta_{1} /\left(\cos ^{2} \beta_{2}-\cos ^{2} \beta_{1}\right)\right] \times
$$

$\left(1+\tan \beta_{1} \tan \vartheta_{1}\right)$

Upon elimination of $\vartheta_{1}$, the relative inflow angle, the propagation speed bécomes simply

$$
\omega R / U=\left[1+a\left(\cos ^{2} \beta_{1} / \cos ^{2} \beta_{2}\right)\right] \csc \beta_{1}
$$

Since $a$ is, in general, a positive quantity, it appears that the influence of discharge angle deviation is to increase the propagation speed over that which would be obtained with a fixed outlet angle, $a=0$.

This value of the constant $A$, which has been obtained in Eq. (38), allows computation of the inlet angle perturbation according to Eq. (22). Thus

$$
\Delta \beta_{1}=\frac{1}{4}\left\{\frac{\frac{\Delta p^{*}}{(1 / 2) \rho U^{2}}+b\left(\beta_{1}-\beta_{1}^{*}\right)}{(\omega R / U)+(b / 4)(1-\alpha)}\right\}\left[F\left(0^{-}, y\right)-\alpha\right]
$$

and using the same reasoning as that employed in the problem of the simple characteristic, the fraction of the blade row periphery which is stalled is 


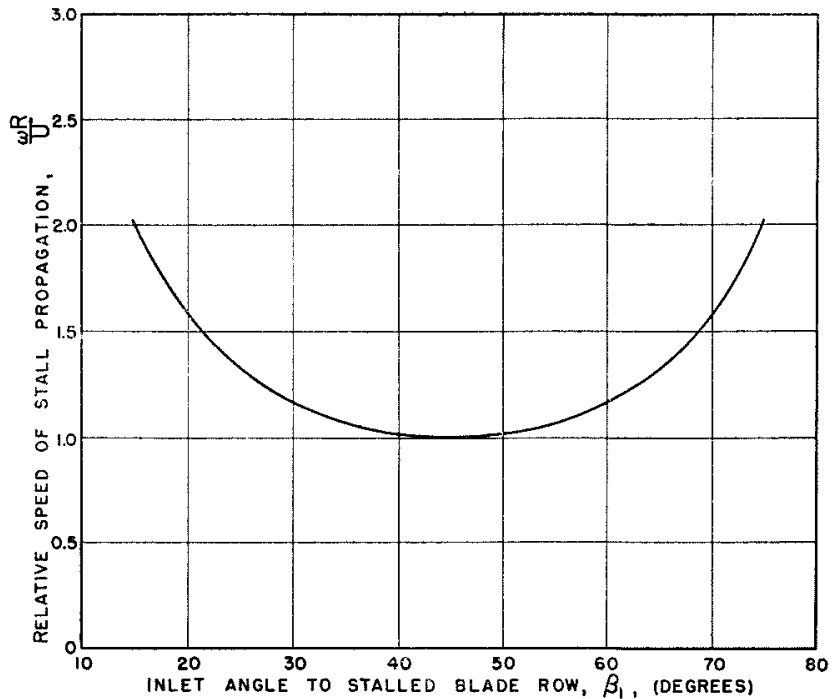

FIG. 10. Propagation speed for simple cascade characteristic.

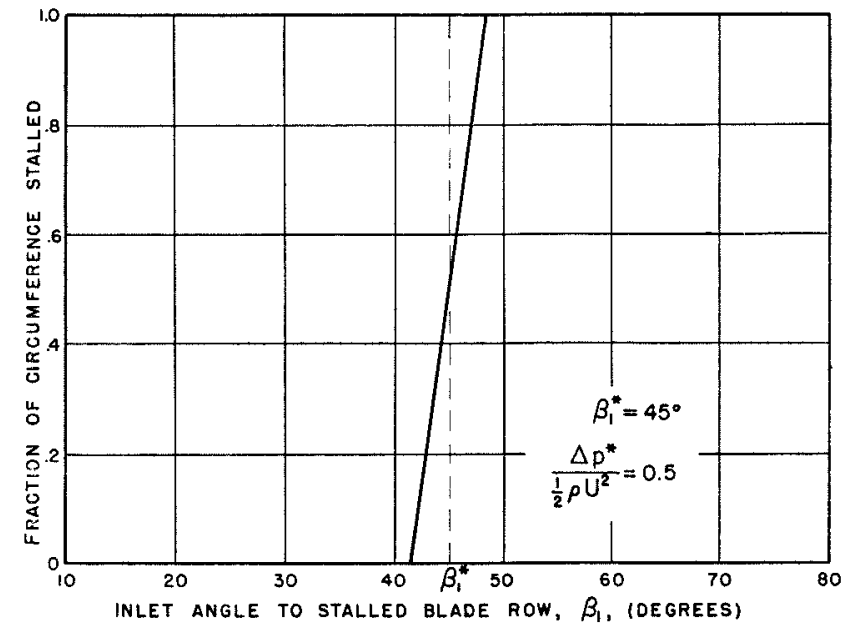

Frg. 11. Variation of length of stalled region with inlet angle for simple cascade characteristic.

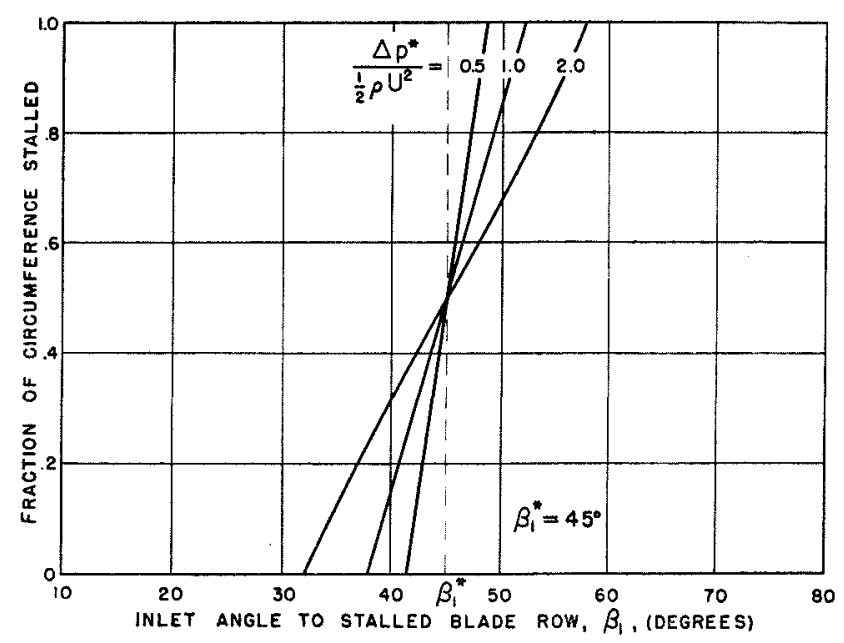

FIG. 12. Variation of length of stalled region with inlet angle for various values of $\Delta p^{*} /\left[(1 / 2) \rho U^{2}\right]=0.5,1.0,2.0$.

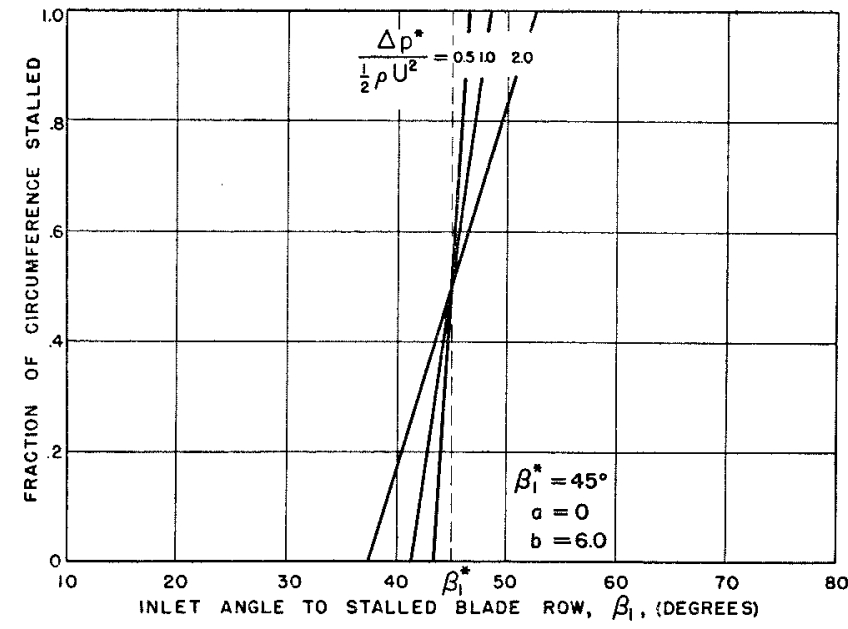

FIG. 13. Effect of more general cascade characteristic upon length of stalled region, $a=0, b=6.0$.

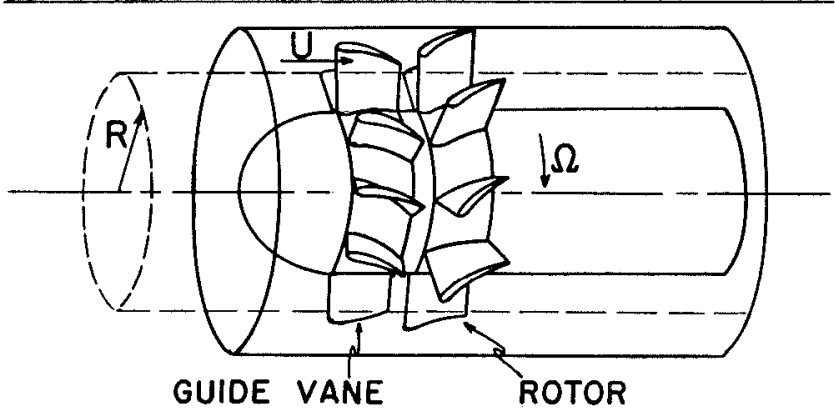

FIG. 14. Diagram of simple axial compressor.
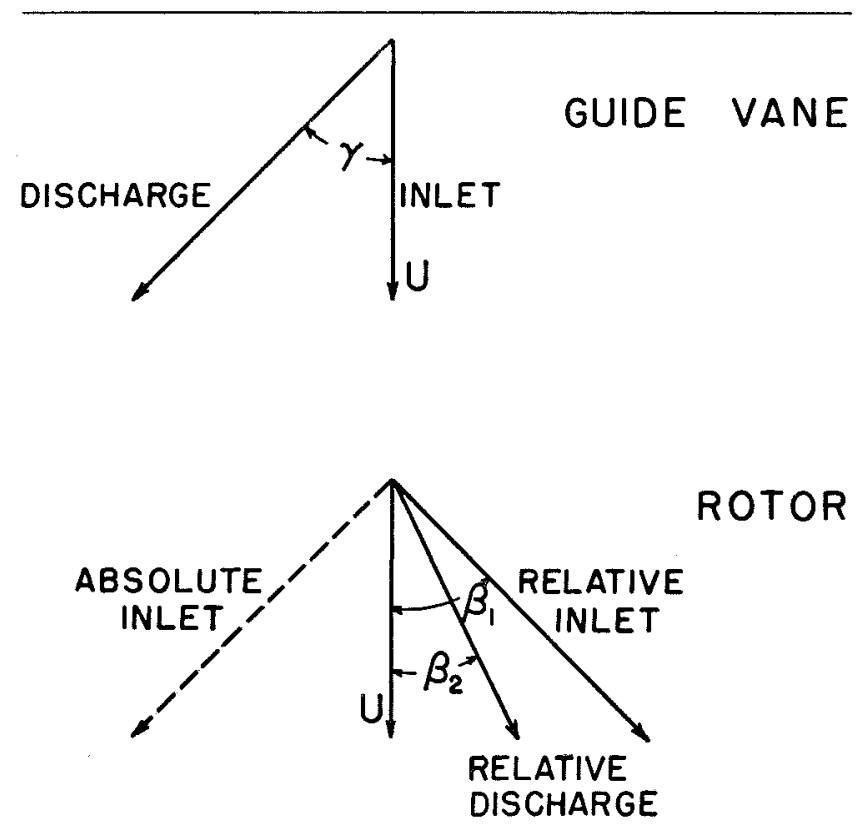

FIG. 15. Velocity diagram for simple compressor stage. 


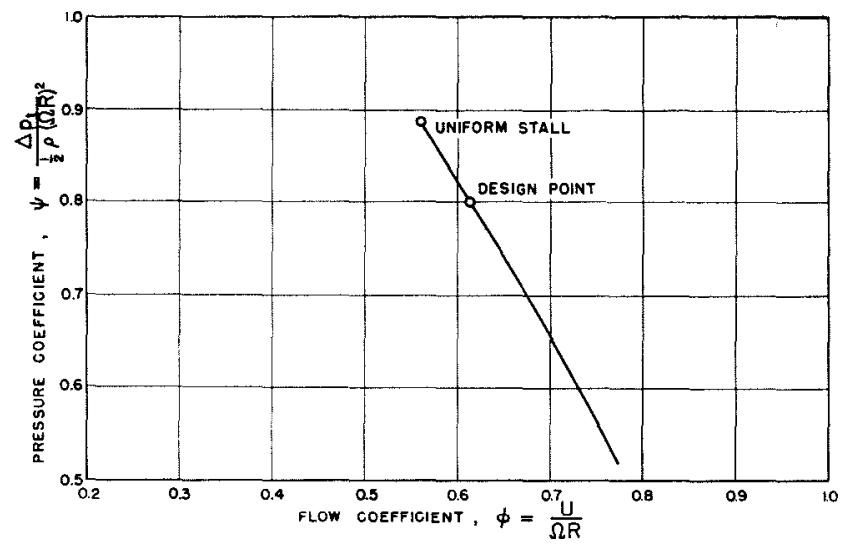

FIG. 16. Normal performance of compressor stage.

$$
\begin{aligned}
& \alpha=\frac{1}{2}-4\left(\beta_{1}^{*}-\beta_{1}\right) \frac{(1 / 2) \rho U^{2}}{\Delta p^{*}} \times \\
& {\left[\left(1+\alpha \frac{\cos ^{2} \beta_{1}}{\cos ^{2} \beta_{2}}\right) \csc 2 \beta_{1}+\frac{b}{8}\right] }
\end{aligned}
$$

where terms of higher order in $\beta_{1}{ }^{*}-\beta_{1}$ have been deleted. Eqs. (39) and (41) give stall propagation speed $\omega R / U$ and the peripheral extent of stall $\alpha$ as functions of $\beta_{1}$ alone for fixed values of $a, b$, and $\Delta p^{*} /\left[(1 / 2) \rho U^{2}\right]$.

Since the value of $a$ is usually small, the variation of outlet angle is not a strong influence on stall propagation speed. Since $\cos ^{2} \beta_{1} / \cos ^{2} \beta_{2}$ is usually less than unity, it is not probable that the propagation speed will be augmented by more than 25 per cent in this manner. The corresponding small influence upon the peripheral extent of the stall region is to decrease the range of inlet angles for which stall propagation may be expected. Similarly the inlet angle where stall propagation will first occur is increased by an increase in the cascade discharge deviation, $a$. The most influential factor in determining the extent of stall regions for a given inlet angle is the cascade pressure rise $\Delta p^{*}$, as was found to be true with the simple cascade characteristic. The influence of the slope of pressure characteristic at the stall is illustrated in Fig. 13 where the variation of $\alpha$ with $\beta_{1}$ is shown for the same values of $\Delta p^{*}(1 / 2) \rho U^{2}$ used in Fig. 12, with the exception that now the slope is taken equal to 6.0. A large slope of the pressure characteristic, such as that shown, may exert considerable influence in reducing the range of inlet angles for which stall propagation is observed.

\section{Performance of a Stalled Compressor Stage}

The results of the analysis of stalling performance of a cascade may be employed to estimate the performance of a simple compressor stage, Fig. 14, which consists of a rotor following an inlet guide vane, which will be considered infinitely far upstream. Then referring to Fig. 15, the absolute outlet angle from the guide vane is $\gamma$. It is convenient to discuss the compressor performance in terms of the two conventional parameters, $\phi$, the flow coefficient, and $\psi$, the pressure coefficient. These are defined as

$$
\begin{gathered}
\phi=U /(\Omega R) \\
\psi=\Delta p_{t} /\left[(1 / 2) \rho(\Omega R)^{2}\right]
\end{gathered}
$$

where $\Omega$ is the angular velocity of the rotor and $\Delta p_{t}$ is the mean increase in total pressure across the stage. Then the compressor characteristic is given by plotting $\psi$ versus $\phi$ as indicated in Fig. 16 where the performance is shown for normal operation up to the stall point that would be reached if all blades stalled simultaneously.

Now according to the velocity diagram the inlet angle $\beta_{1}$ to the rotor is

$$
\tan \beta_{1}=(1 / \phi)-\tan \gamma
$$

so that the value $\phi^{*}$ of the flow coefficient for which the normal stall occurs is $\phi^{*}=\left(\tan \beta_{1}+\tan \gamma\right)^{-1}$. Furthermore, the total pressure rise $\Delta p_{t}$ across the rotor is, for no losses in the blade row,

$$
\Delta p_{t}=\rho \Omega R\left(U \tan \beta_{1}-U \tan \beta_{2}\right)
$$

or in terms of the pressure and flow coefficients

$$
\psi=2 \phi\left(\tan \beta_{1}-\tan \beta_{2}\right)
$$

which holds up to the stall angle. Thus specification of the flow coefficient and pressure coefficient is equivalent to specifying the inlet and discharge angles from the rotor.

Referring to the compressor performance of Fig. 16, it is clear that, as the flow coefficient $\phi$ is reduced, a point is reached where stall propagation is first possible. This is just the condition that $\alpha=0$ and, assuming for the moment the simplest cascade characteristic where $a=b=0$, it follows from Eq. (30) that

$$
\Delta p^{*} /\left[(1 / 2) \rho U^{2}\right]=8\left(\beta_{1}^{*}-\beta_{1}\right) \csc 2 \beta_{1}
$$

Now according to Eq. (44) $\beta_{1}{ }^{*}-\beta_{1} \approx\left(\cos ^{2} \beta_{1} / \phi^{2}\right)$ $\left(\phi-\phi^{*}\right)$, and, consequently, the value $\phi_{i}$ of the flow coefficient for which stall propagation first appears is

$$
\phi_{i}=\phi^{*}\left[1+\frac{1}{4}\left(1-\phi^{*} \tan \gamma\right) \frac{\Delta p^{*}}{(1 / 2) \rho U^{2}}\right]
$$

Actually, of course, the stall can not start with a region of arbitrarily small peripheral extent but must involve at least one blade channel. Under usual circumstances this represents a negligible influence on $\phi_{i}$.

The total pressure loss involved due to the propagating stalled region is simply equal to the static pressure perturbation far downstream of the blade row, the change in kinetic energy resulting from distortion of the flow field being of second order. Thus, according to Eq. (18), the pressure field perturbation far downstream is $P_{2}(\infty)=-\alpha\left\{\Delta p^{*} /\left[(1 / 2) \rho U^{2}\right]\right\}$ and hence the change $\Delta \psi$ in pressure coefficient due to stall is just

$$
\Delta \psi \approx-\alpha \phi^{2}\left\{\Delta p /\left[(1 / 2) \rho U^{2}\right]\right\}
$$

The pressure loss is thus simply proportional to the fraction $\alpha$ of the circumference which is stalled, so that utilizing Eq. (30) of the previous calculation 


$$
\Delta \psi=\left[-2 \phi^{*} /\left(1-\phi^{*} \tan \gamma\right)\right]\left(\phi_{i}-\phi\right)
$$

Therefore, as the compressor stage is throttled, stall propagation first becomes possible at a flow coefficient $\phi_{i}$; as the flow coefficient is reduced farther, the pressure coefficient falls below that which would occur in the absence of stall by an amount proportional to the reduction of the flow coefficient below $\phi_{i}$. To the present approximation this reduction continues until the periphery is entirely stalled - that is, $\alpha=1$, at which point the loss in pressure coefficient is simply

$$
\phi^{2}\left\{\Delta p^{*} /\left[(1 / 2) \rho U^{2}\right]\right\}
$$

Actually the small perturbation analysis employed is invalid when $\alpha$ becomes of appreciable size, and losses computed under these circumstances are at best of the proper magnitude.

For the general cascade characteristic the flow coefficient for incipient propagating stall is

$$
\begin{aligned}
\phi_{i}=\phi^{*}\left(1+\left\{\left[\frac{1}{4}\left(1-\phi^{*} \tan \gamma\right) \frac{\Delta p^{*}}{(1 / 2) \rho U^{2}}\right] \div\right.\right. \\
\left.\left.\left(1+a \frac{\cos ^{2} \beta_{1}{ }^{*}}{\cos ^{2} \beta_{2}}+\frac{9}{32} b \sin ^{2} \beta_{1}{ }^{*}\right)\right\}\right)
\end{aligned}
$$

while the change in pressure coefficient $\Delta \psi$ due to stall becomes, neglecting terms of small order,

$$
\begin{aligned}
& \Delta \psi=-\left[\frac{2 \phi^{*}}{1-\phi^{*} \tan \gamma}\left(1+a \frac{\cos ^{2} \beta_{1}{ }^{*}}{\cos ^{2} \beta_{2}{ }^{*}}\right)+\right. \\
& \left.\frac{b}{2\left(1+\tan ^{2} \beta_{1}^{*}\right)}\right]
\end{aligned}
$$

The principle effect of variations in pressure rise and cascade discharge angle due to changing inlet angle is, under normal circumstances, to increase the pressure loss somewhat.

To illustrate the influence of propagating stall upon the compressor performance, consider the arbitrary compressor characteristic given in Fig. 16. Under conditions of uniform flow the stage stalls at a rotor inlet angle of $\beta_{1} \equiv \beta_{1}{ }^{*}=51^{\circ} 30^{\prime}$ and at a corresponding flow coefficient $\phi=\phi^{*}=0.56$. Assume further that the guide vane discharge angle is $\gamma=28^{\circ}$, while $a=0.25$ and $b=6.5$. The parameters employed are approxi-

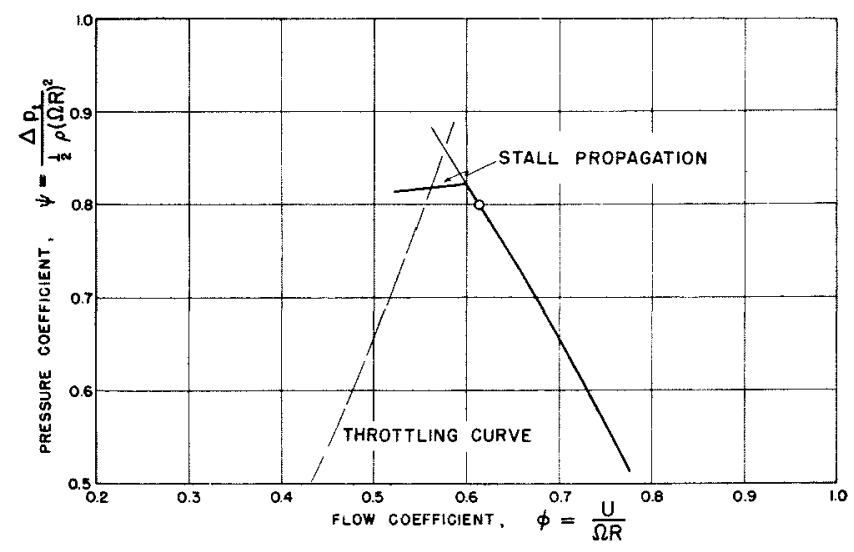

FIG. 17. Performance of compressor stage with stall propagation.

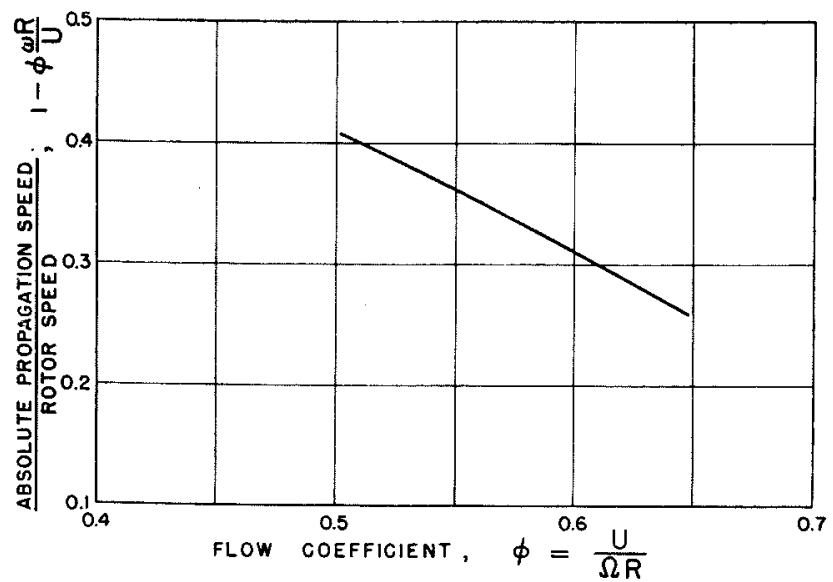

FIG. 18. Rate of stall propagation for compressor stage.

mately those values for the guide vane and first rotor near the root of the compressor employed in the experiments of Rannie and Iura using vortex blading. As the compressor is throttled, the first possibility of stall propagation occurs, according to Eq. (49), at $\phi_{i}=0.6$. Then as the flow is further reduced, the pressure coefficient drops below the normal characteristic by an amount that is easily calculated from Eq. (50). The resulting performance with propagating stall is shown as a broken line in Fig. 17.

Now suppose, as is usually the case, the flow through the compressor is reduced by adjusting a throttle downstream of the compressor while the compressor speed is held constant. Then the pressure rise $\Delta p_{t}$ imposed upon the compressor is proportional to $U^{2}$, or in terms of the compressor parameters $\psi=\kappa \phi^{2}$, where the coefficient $\kappa$ depends upon the throttle setting. Therefore the states of normal operation and propagating stall which correspond to the same throttle setting lie along a parabolic "throttling" curve such as that indicated in Fig. 17. It is probable, there being some indication from the experiments of Rannie and Iura, that the stall propagation does not appear as soon as the flow coefficient $\phi_{i}$ is encountered, but, rather, the transition from normal operation to stall propagation takes place as the result of instability of the former at a flow coefficient somewhat less than $\phi_{i}$. In this case the transition must be to that state of stall propagation connected by the appropriate throttling curve.

The values of stall propagation speed for the compressor stage follow directly from Eq. (39) using the relation between flow coefficient and rotor inlet angle given by Eq. (44). Since the speeds of propagation are usually observed relative to the fixed blade rows, the significant quantity is $(\Omega-\omega) R$, and the ratio of absolute angular velocity of stall propagation to the rotor angular velocity is just

$$
1-\phi(\omega R / U)
$$

This speed of propagation for the compressor stage is shown in Fig. 18 at the performance conditions of Fig. 17. The stall propagation speed observed by Rannie and Iura is about 0.3 , and it is of interest that the value of propagation speed computed near the onset of stall- 
that is, at $\phi=0.6$-is in remarkable agreement with their value. In view of the facts that a two-dimensional theory has been employed and that the interference of neither the guide vanes nor the stalled blade rows downstream of the first rotor is accounted for, the agreement must be considered, in a certain measure, fortuitous. Although the magnitude is essentially correct, the only discrepancy seems to be the variation of calculated propagation speed with flow coefficient, while the experimental value appears constant over a wide range. This feature, however, is simply a result of the linearization. For whereas in the linearized analysis the flow approaches the stalling blades at nearly the undisturbed upstream flow angle, the real flow approaching the blades is strongly disturbed by stall and seems to adjust itself to almost a constant flow angle, regardless of how severely the compressor is throttled. Consequently, as was clear at the outset, accuracy of the calculation can be expected only near the condition of incipient stall.

\section{CONCLUdING REMARKS}

From the experimental data that are available at the present time, it appears that the model upon which the foregoing analysis is based is a realistic one and contains the essence of the phenomena that have been observed. Based upon this model, the features of stall propagation - that is, the extent of stall regionthe pressure loss associated with stalled operation together with its attending effect upon performance, as well as the speed of stall propagation arise naturally and simply. Discrepancies in comparison with experimental results on propagation speed cannot be interpreted as inadequacy of the model until either a more detailed experimental investigation of the mechanism is undertaken or the results are extended to situations more nearly approximating those on which observations have been made. Extensions appear logical in two directions: the effect of mutual interference between adjacent blade rows in relative motion and stall propagation in a three-dimensional actuator desk.

\section{REFERENCES}

${ }^{1}$ Emmons, H. W., Pearson, C. E., and Grant, H. P., Compressor Surge and Stall Propagation, Presented at Annual Meeting, American Society of Mechanical Engineers, New York, December, 1953.

${ }^{2}$ Iura, T., and Rannie, W. D., Experimental Investigation of Propagating Stall in Axial Flciv Compressors, Paper No. 53-SA69, American Society of Mechanical Engineers, presented in Los Angeles, July 29, 1953 .

${ }^{3}$ Huppert, M. C., and Benser, W. A., Some Stall and Surge Phenomena in Axial Flow Compressors, Presented at TwentyFirst Annual Meeting, Institute of the Aeronautical Sciences, New York, January, 1953.

"Sears, W. R., On Asymmetric Flow in an Axial-Flow Compressor Stage, Journal of Applied Mechanics, pp. 57-62, March, 1953.

"Sears, W. R., A Theory of "Rotating Stall" in Axial-Flow Compressors, Report in Partial Fulfillment of Contract AF 33(038)-21406, Office of Scientific Research, U.S. Air Force.

' Lamb, Sir Horace, Hydrodynamics, Sixth Edition, p. 71, Cambridge University Press, 1932. 